\title{
Variação da fluência da fala em idosos***
}

\author{
Speech fluency variation in elderly
}

\author{
Claudia Regina Furquim de Andrade * \\ Vanessa de Oliveira Martins**
}

\begin{abstract}
*Fonoaudióloga. Professora Titular do Departamento de Fisioterapia, Fonoaudiologia e Terapia Ocupacional da Faculdade de Medicina da Universidade de São Paulo (FMUSP). Endereço para correspondência: Rua Cipotânea, 51 - São Paulo - SP CEP 05360-160 (clauan@usp.br)

**Fonoaudióloga. Doutora em Semiótica e Linguística Geral pela Faculdade de Filosofia, Letras e Ciências Humanas da Universidade de São Paulo (USP).

***Trabalho Realizado no Departamento de Fisioterapia, Fonoaudiologia e Terapia Ocupacional da FMUSP.
\end{abstract}

Artigo Original de Pesquisa

Artigo Submetido a Avaliação por Pares

Conflito de Interesse: não

\section{Abstract}

Background: a number of changes in the speech of normally aging adults have been described in the literature. However age-related changes in fluency have received little attention. Aim: to verify the Fluency Profile of elderlies regarding all of the different fluency parameters. Method: participants were 128 elderlies, of both genders, aged above 60 years. Speech samples of all participants were gathered. They were analyzed according to: type of speech disruption; speech rate and frequency of speech disruptions, based on the analyses of 200 fluent syllables. Participants were compared between themselves regarding the decades of life. Individuals with ages above 80 years constituted a single group. Results: statistical analysis indicated significant differences between the decades only for syllables per minute. For the group of individuals with ages above 80 years, statistical significance was observed indicating an increase in the number of speech disruptions and a decrease in speech rate. Conclusion: regarding the fluency parameters analyzed in this study, the effect of aging seems to be more expressive after the age of 80 years.

Key Words: Aged; Speech; Fluency; Methods.

\section{Resumo}

Tema: tem sido descrito na literatura a existência de mudanças na fala decorrentes do envelhecimento, entretanto, nesses estudos, as variações da fluência têm recebido pouca atenção. Objetivo: verificar o perfil da fluência da fala em idosos em diferentes parâmetros. Método: os participantes foram 128 indivíduos com idade acima de 60 anos, de ambos os gêneros. Foram obtidas amostras de fala de todos os participantes e analisadas segundo as variáveis de: tipos de rupturas; velocidade de fala e frequência de rupturas. A análise computou 200 sílabas fluentes da amostra. Resultados: a análise estatística mostrou diferenças estatisticamente significantes entre as décadas somente para a variável de sílabas por minuto. No grupo acima de 80 anos houve significância estatística indicando aumento das rupturas de fala e decréscimo da velocidade. Conclusão: o efeito da idade parece ser mais expressivo depois dos oitenta anos em relação aos parâmetros de fluência da fala analisados nesse estudo.

Palavras-Chave: Idosos; Fala; Fluência; Métodos. 


\section{Introdução}

O estudo da influência da idade sobre a comunicação se torna importante em decorrência do aumento da expectativa de vida em quase todos os países (desenvolvidos e em desenvolvimento, incluindo o Brasil). Ataxa proporcional de envelhecimento (acima de 60 anos) $)^{1}$ tem crescido progressivamente.

De acordo com o IBGE, estima-se que a população de idosos em 2020 será em torno de 28 milhões de pessoas e em 2040 em torno de 52 milhões.

Durante o processo de envelhecimento, algumas mudanças ocorrem e estão relacionadas com: precisão, fluência, qualidade vocal e efetividade comunicativa ${ }^{2}$. Essas mudanças parecem ser similares àquelas ocorridas em diversas doenças, comumente observadas na população de idosos. Dados normativos sobre as variáveis da fala decorrentes do envelhecimento populacional são de grande importância. A demanda cognitiva, sensorial e motora para a produção da fala parecem estar relacionadas com os processos típicos do envelhecimento e com as doenças do envelhecimento ${ }^{2}$.

Em acordo com o paradigma teórico adotado nesse estudo, a fluência é o fluxo contínuo e suave de produção da fala ${ }^{3}$. As rupturas do fluxo da fala podem ser naturais ou patológicas, dependendo de um complexo processo neurofuncional e linguístico, que é responsável pela temporalização dos sons na construção das palavras e sentenças ${ }^{4-6}$. Os níveis de fluência e disfluência podem ser medidos segundo as variáveis de tipologia das rupturas, velocidade de fala e frequência das rupturas ${ }^{5,7}$.

Alguns pesquisadores associam a lentidão da taxa articulatória ao envelhecimento ${ }^{8-12}$, entretanto a taxa de disfluências não parece vulnerável ao processo do envelhecimento ${ }^{8-14}$.

Para Pretti ${ }^{15}$, o traço de maior saliência na fluência do idoso são as rupturas, que decorreriam dos aspectos prosódicos e da própria organização do discurso. $\mathrm{O}$ autor aponta que a descontinuidade da fala é um fenômeno regular da linguagem oral, em qualquer idade. Essa descontinuidade é marcada por rupturas que podem ocorrer nos níveis: pragmático (descontinuidade de tópico, interferências de segmentos); sintático (descontinuidade na sentença); lexical (hesitações e termos truncados) e fonológico (presença de pausas).

As variações da fluência relacionadas com a idade têm recebido pouca atenção dos pesquisadores, principalmente para os idosos acima de 90 anos, embora haja um consenso que estudos das variações da fluência, ao longo da vida, sejam necessários ${ }^{10-11,14,16}$. Estudos das variações da fluência, típicas da infância, poderiam contribuir para a melhor compreensão dos parâmetros de fala e da influência do envelhecimento sobre eles ${ }^{12}$.

O objetivo dessa pesquisa foi o levantamento das especificidades do perfil da fluência da fala (tipologia das rupturas, velocidade de fala e frequência das rupturas) ${ }^{5}$ em indivíduos idosos. A hipótese do estudo é que existe a influência do fator idade (variabilidade nas tipologias das rupturas, redução da velocidade de fala e aumento da taxa de rupturas) na população estudada.

\section{Método}

Os dados foram colhidos somente após os procedimentos éticos pertinentes. O estudo foi aprovado pela comissão de ética institucional (CAPPesq do Hospital das Clínicas da Faculdade de Medicina da Universidade de São Paulo HCFMUSP - número 848/01) e os termos de consentimento obtidos para todos os participantes.

Os participantes do estudo foram 128 idosos, de ambos os gêneros, com idade acima de 60 anos. Todos os participantes eram residentes na Cidade de São Paulo. Para as comparações, os participantes foram divididos por faixa etária: (a) 60-69 anos (n = 36); (b) 70-79 anos ( $\mathrm{n}=48)$; (c) 80-89 anos ( $\mathrm{n}=31$ ); (d) 90-99 anos $(n=13)$. Em todos os grupos houve predominância do gênero feminino, sendo: $69 \%$ aos 60-69 anos, 73\% aos 70-79 anos, 58\% aos 80-89 anos e, 85\% aos 90-99 anos. Essa diferença é compatível com a variação populacional.

Os critérios gerais para a inclusão dos participantes foram: ausência de antecedentes familiares para a gagueira; ausência de queixas de gagueira; ausência de doenças crônicas; screening negativo para distúrbios da comunicação (linguagem, audição, cognição etc.). Para esse screening foi utilizado um protocolo simples durante a primeira entrevista. Os critérios específicos para a inclusão dos participantes foram baseados no Stuttering Severity Instrument (SSI-3) $)^{17}$.

A metodologia usada para coletar e analisar as amostras de fala são as propostas no Protocolo de Avaliação da Fluência da Fala ${ }^{7}$, que considera, em 200 sílabas expressas (livres de rupturas), os seguintes parâmetros: tipologia das rupturas (disfluências comuns e gagas); velocidade de fala (palavras e sílabas por minuto) e frequência das rupturas (porcentagem de descontinuidade de fala e porcentagem de disfluências gagas).

As disfluências comuns são: hesitações; interjeições; revisões; palavras não terminadas; repetições de palavras, de segmentos e de frases. As disfluências gagas são: repetições de sílabas; repetições de sons; prolongamentos; bloqueios; pausas (mais de dois segundos) e intrusões de sons ou segmentos. 
Para a obtenção das sílabas por minuto, foram consideradas, para cada participante, o total de 200 sílabas expressas da emissão, dividida pelo tempo total da amostra. Para a medida, foi utilizado um cronômetro digital ${ }^{10}$. As palavras e sílabas por minuto foram obtidas pelo cálculo do número total de palavras/sílabas da emissão divididas pelo tempo total da amostra. A frequência de rupturas considerou a porcentagem total de rupturas pelo tempo da amostra.

Para a análise estatística foi utilizado o teste de ANOVA visando comparar as décadas entre si, para todas as variáveis. O teste de Tukey foi utilizado nas comparações múltiplas. O nível de significância adotado foi de $0,5 \%$.

\section{Resultados}

A Tabela 1 mostra a média e o desvio padrão, por faixa etária, para as variáveis do perfil da fluência da fala. Segundo os resultados do teste de ANOVA, houve diferença estatisticamente significante somente na variável de sílabas por minuto. O teste de Tukey não mostrou diferenças significantes para as variáveis testadas.
Para uma melhor interpretação dos dados, os participantes foram agrupados em: GI (60 a 79 anos) com um n = 84 e GII (acima de 80 anos) com um n = 44. Esse agrupamento etário seguiu a proposta do Medical Subject Headings (Index Medicus) ${ }^{18}$. A média de idade para GI foi de 70,19 anos (DP=5.47) e para GII foi de 86,43 anos (DP = 5.05). A distribuição dos gêneros foi a mesma para ambos os grupos $\left(\mathrm{X}^{2}=0,415 ; \mathrm{Gl} .=1 ; \mathrm{p}=0,519\right)$. Amédia de idade para o gênero feminino foi de 75,82 anos (DP $=9,78$ ) e para o gênero masculino foi de 75,67 anos $(\mathrm{DP}=8,56)$ Não tendo sido obtida diferença estatisticamente significativa.

A Tabela 2 compara as variáveis para GI e GII, foram obtidas diferenças estatisticamente significantes para todas as variáveis, exceto para as disfluências gagas. Esse dado aponta que em GII houve um aumento no número de rupturas comuns e um aumento na porcentagem geral de rupturas. Foi observado para GII um decréscimo na velocidade de fala (tanto para palavras quanto para sílabas por minuto).

TABELA 1. Resultados das variáveis do perfil da fluência da fala nas faixas etárias pesquisadas.

\begin{tabular}{|c|c|c|c|c|c|}
\hline & Década de Vida & Média & DP & F & $\mathrm{p}$ \\
\hline \multirow[t]{4}{*}{ DC } & 60 & 11,75 & 6,49 & \multirow{4}{*}{1,91} & \multirow{4}{*}{0,131} \\
\hline & 70 & 10,98 & 6,45 & & \\
\hline & 80 & 13,58 & 5,9 & & \\
\hline & 90 & 15,39 & 10,54 & & \\
\hline \multirow[t]{4}{*}{ DGG } & 60 & 2,17 & 2,1 & \multirow{4}{*}{0,65} & \multirow{4}{*}{0,587} \\
\hline & 70 & 1,79 & 2,58 & & \\
\hline & 80 & 2,55 & 2,29 & & \\
\hline & 90 & 2,15 & 2,51 & & \\
\hline \multirow[t]{5}{*}{$\mathrm{P} / \mathrm{M}$} & 60 & 118,4 & 29,26 & \multirow{4}{*}{2,09} & \multirow{4}{*}{0,105} \\
\hline & 70 & 111,38 & 30,44 & & \\
\hline & 80 & 102,92 & 30,10 & & \\
\hline & 90 & 99,67 & 28,14 & & \\
\hline & 60 & 216,94 & 53,24 & \multirow{4}{*}{3,01} & \multirow{4}{*}{$0,033^{*}$} \\
\hline \multirow{4}{*}{$\mathrm{S} / \mathrm{M}$} & 70 & 201,64 & 52,4 & & \\
\hline & 80 & 183,61 & 54,56 & & \\
\hline & 90 & 177,34 & 50,53 & & \\
\hline & 60 & 6,96 & 3,72 & \multirow{4}{*}{1,96} & \multirow{4}{*}{0,123} \\
\hline \multirow{3}{*}{$\% \mathrm{TR}$} & 70 & 6,27 & 3,7 & & \\
\hline & 80 & 8,0 & 3,58 & & \\
\hline & 90 & 8,73 & 6,14 & & \\
\hline
\end{tabular}

Legenda: DP = desvio-padrão; $\mathrm{DC}=$ disfluências comuns; $\mathrm{DGG}=$ disfluências gagas; $\mathrm{P} / \mathrm{M}=$ palavras por minuto; $\mathrm{S} / \mathrm{M}=$ sílabas por minuto; $\mathrm{TR}=$ taxa de rupturas. 
TABELA 2. Resultados para as variáveis do Perfil da Fluência da Fala na comparação GI/GII.

\begin{tabular}{|c|c|c|c|c|c|}
\hline & Grupo & Média & DP & $\mathrm{F}$ & $\mathrm{p}$ \\
\hline \multirow[t]{2}{*}{ DC } & GI & 11,31 & 6,44 & \multirow{2}{*}{4,89} & \multirow{2}{*}{$0,029 *$} \\
\hline & GII & 14,11 & 7,48 & & \\
\hline \multirow[t]{2}{*}{ DGG } & GI & 1,95 & 2,38 & \multirow{2}{*}{1,19} & \multirow{2}{*}{0,278} \\
\hline & GII & 2,43 & 2,34 & & \\
\hline \multirow[t]{2}{*}{$\mathrm{P} / \mathrm{M}$} & GI & 114,4 & 30,0 & \multirow{2}{*}{5,05} & \multirow{2}{*}{$0,026^{*}$} \\
\hline & GII & 102,0 & 29,2 & & \\
\hline \multirow[t]{2}{*}{$\mathrm{S} / \mathrm{M}$} & GI & 208,2 & 53,0 & \multirow{2}{*}{7,19} & \multirow{2}{*}{$0,008^{*}$} \\
\hline & GII & 181,8 & 52,9 & & \\
\hline \multirow[t]{2}{*}{ \%TR } & GI & 6,57 & 3,7 & \multirow{2}{*}{5,01} & \multirow{2}{*}{$0,027^{*}$} \\
\hline & GII & 8,22 & 4,42 & & \\
\hline
\end{tabular}

Legenda: DP = desvio-padrão; $\mathrm{DC}$ = disfluências comuns; $\mathrm{DGG}=$ disfluências gagas; $\mathrm{P} / \mathrm{M}$ = palavras por minuto; $\mathrm{S} / \mathrm{M}=$ sílabas por minuto; $\mathrm{TR}=$ taxa de rupturas.

Para melhor compreensão dos resultados obtidos, a Tabela 3 apresenta a análise isolada das tipologias das disfluências. As tipologias que diferenciaram os grupos foram as hesitações e as pausas, ambas significantemente mais ocorrentes em GII.

TABELA 3. Comparação das tipologias de ruptura entre GI e GII.

\begin{tabular}{|c|c|c|c|c|c|}
\hline & Grupo & Média & $\mathrm{DP}$ & $\mathrm{F}$ & $\mathrm{p}$ \\
\hline hesitação & $\begin{array}{c}\text { GI } \\
\text { GII }\end{array}$ & $\begin{array}{l}2,45 \\
4,11\end{array}$ & $\begin{array}{c}2,45 \\
3,3\end{array}$ & 10,4 & $0,002 *$ \\
\hline interjeição & $\begin{array}{c}\text { GI } \\
\text { GII }\end{array}$ & $\begin{array}{l}4,31 \\
5,50\end{array}$ & $\begin{array}{l}4,12 \\
4,89\end{array}$ & 2,12 & 0,148 \\
\hline revisão & $\begin{array}{c}\text { GI } \\
\text { GII }\end{array}$ & $\begin{array}{l}1,85 \\
1,66\end{array}$ & $\begin{array}{l}1,58 \\
1,43\end{array}$ & 0,43 & 0,514 \\
\hline repetição de palavras & $\begin{array}{c}\text { GI } \\
\text { GII }\end{array}$ & $\begin{array}{l}1,88 \\
1,77\end{array}$ & $\begin{array}{l}1,65 \\
1,41\end{array}$ & 0,14 & 0,713 \\
\hline palavras não terminadas & $\begin{array}{c}\text { GI } \\
\text { GII }\end{array}$ & $\begin{array}{l}0,381 \\
0,318\end{array}$ & $\begin{array}{l}0,775 \\
0,518\end{array}$ & 0,23 & 0,630 \\
\hline repetição de segmentos & $\begin{array}{c}\text { GI } \\
\text { GII }\end{array}$ & $\begin{array}{c}0,429 \\
0,70\end{array}$ & $\begin{array}{c}0,811 \\
1,30\end{array}$ & 2,17 & 0,143 \\
\hline repetição de sílabas & $\begin{array}{c}\text { GI } \\
\text { GII }\end{array}$ & $\begin{array}{c}0,25 \\
0,295\end{array}$ & $\begin{array}{l}0,557 \\
0,632\end{array}$ & 0,18 & 0,676 \\
\hline prolongamentos & $\begin{array}{l}\text { GI } \\
\text { GII }\end{array}$ & $\begin{array}{l}1,20 \\
0,82\end{array}$ & $\begin{array}{l}1,38 \\
1,35\end{array}$ & 2,27 & 0,134 \\
\hline pausas & $\begin{array}{c}\text { GI } \\
\text { GII }\end{array}$ & $\begin{array}{l}0,40 \\
1,18\end{array}$ & $\begin{array}{l}1,20 \\
1,92\end{array}$ & 7,88 & $0,006^{*}$ \\
\hline
\end{tabular}

Legenda:DP = desvio-padrão.

\section{Discussão}

O estudo aqui apresentado teve como objetivo verificar as especificidades do perfil da fluência da fala em idosos saudáveis. A contribuição dessa pesquisa é permitir a melhor compreensão das variações da fluência da fala geradas pelo processo de envelhecimento. Com o aumento da expectativa de vida, é importante conhecer as especificidades da comunicação desse grupo de clientes, que se tornam de maior risco de doenças crônicas, hospitalizações e institucionalizações ${ }^{19}$. É importante que o fonoaudiólogo conheça o padrão da fluência da fala esperado no envelhecimento normal, para que possa distinguir, com maior segurança, os processos de fala desviantes. 
Ramose et al. ${ }^{20-21}$ consideram que a maior parte dos idosos que apresenta pelo menos uma doença crônica pode ser considerada saudável se não apresentarem sequelas ou restrições nas habilidades diárias. Para os autores, a autonomia é o que está em risco com o envelhecimento. Os autores consideram autonomia a capacidade de determinar e executar suas próprias tarefas. Nesse contexto, a habilidade comunicativa ocupa uma importante face do processo, sendo relevante a realização de estudos sobre a variabilidade do envelhecimento saudável nos aspectos comunicativos.

No estudo aqui apresentado, quando comparadas as diferentes décadas de vida, não houve diferenciação para as tipologias das disfluências e para a porcentagem de total de rupturas. Foi observado que, ao longo das décadas, existe um decréscimo na velocidade de fala tanto em palavras quanto em sílabas por minuto, embora tenha havido significância estatística somente para sílabas por minuto. O teste utilizado não localizou em que década isso ocorreu. Esses dados podem ser indicativos que a transmissão da informação, em idosos saudáveis, não se diferencia significativamente ao longo das décadas. A diferença observada na velocidade articulatória (sílabas / minuto) deve ser melhor pesquisada, pois pode ser decorrente tanto das condições bucais (próteses dentárias mal adaptadas) quanto, realmente, de déficits no processamento motor oral ou fonológico.

A comparação entre os idosos abaixo (GI) e acima de 80 anos (GII) demonstra uma diferença estatisticamente significante para todas as variáveis pesquisadas, exceto para aquelas sugestivas de gagueira. É importante mencionar que não seria esperado que com o envelhecimento surgissem rupturas indicativas da patologia da gagueira. Não desconsiderando possíveis distorções amostrais, podemos observar a existência de uma variabilidade na fluência da fala entre idosos acima e abaixo dos 80 anos. Para o grupo acima dos 80 anos houve um aumento das hesitações e pausas e uma redução da velocidade de fala, tanto em palavras por minuto (quantidade de mensagem transmitida) quanto em sílabas por minuto (habilidade motora de co-articulação).

Para ambos os grupos, a tipologia de maior ocorrência foi a interjeição, corroborando com estudos prévios ${ }^{10-22-23}$, lembrando que as únicas disfluências encontradas com diferença estatisticamente significante em nosso estudo foram as hesitações e pausas, com maior ocorrência nos idosos acima de 80 anos.

Para Pretti ${ }^{15}$, a fala dos idosos acima dos 80 anos apresenta o excesso de pausas determina um ritmo construído pela emissão de segmentos curtos, pronunciados rapidamente, que levam a perda da força no final da palavra, tornando os sons ininteligíveis e gerando no ouvinte a impressão de cansaço. Para o autor, as disfluências transmitem uma sensação de insegurança que pode ser reconhecida como uma característica marcante da fala das pessoas idosas. As pausas podem ocorrer em posições inesperadas da emissão e as hesitações podem ser causadas por falhas da memória ou pela incerteza do que será dito. Ainda segundo o autor, com o avanço da idade, as pausas tendem a aumentar e a duração da articulação tende a diminuir, indicando que os idosos acima de 80 anos apresentam uma fragilidade não só nos aspectos motores mas também nos aspectos cognitivos e de fala.

A pesquisa aqui apresentada tem grande importância em termos de generalização, pelo representativo número de participantes, podendo ter seus resultados considerados como um modelo de tipicidade dos padrões da fluência em idosos. Esses resultados podem servir como parâmetros para diagnóstico e controle da efetividade dos tratamentos.

Outra colaboração do estudo está relacionada com a variável da Língua. Estudos experimentais sobre a fluência da fala em idosos refletem os achados para os falantes do Inglês. Estudos que usem valores de referência próprios de cada Língua são de grande importância para o correto diagnóstico ${ }^{24}$.

\section{Conclusão}

Os resultados do estudo indicam que houve a confirmação parcial da hipótese apresentada. Não foram obtidos resultados significantes para as variações da fluência quando observadas as décadas de envelhecimento. Quando agrupadas as décadas em idosos acima e abaixo de 80 anos, a hipótese do estudo foi confirmada, indicando haver modificações na fluência da fala em decorrência do envelhecimento. 


\section{Referências Bibliográficas}

1. Brasil, Ministério da Saúde, Secretaria de Atenção à Saúde, Departamento de Atenção Básica. Envelhecimento e saúde da pessoa idosa. Brasília: Ministério da Saúde, 2006. 192p. (Série A - Normas e Manuais Técnicos) (Cadernos de Atenção Básica, $n^{\circ}$ 19).

2. Kent RD. Research on speech motor control and its disorders: a review and prospective. J Commun Disord 2000;33:391-428.

3. Starkweather CW, Givens-Ackerman J. Stuttering. Austin: PRO-ED; 1997.

4. Perkins W, Kent RD, Curlee RF. A Theory of neuropsycholinguistic function in stuttering. J Speech Hear Res. 1991;34(4):734-52.

5. Andrade CRF. Programa de promoção da fluência para adultos - aplicações diferenciadas. [Tese de Livre-Docência]. São Paulo: Faculdade de Medicina da Universidade de São Paulo; 1999.

6. Andrade CRF. Abordagem neurolingüística e motora da gagueira. In: Ferreira LP, Befi-Lopes DM, Limonge SCO (eds). Tratado de Fonoaudiologia. São Paulo: Roca; 2004. p. 1001-26.

7. Andrade CRF de. Protocolo para avaliação da fluência da fala. Pró-Fono. 2000;12(2):131-4.

8. Mysak EE. Pitch and duration characteristics of older males. J Speech Hear Res. 1959;9:273-7.

9. Ryan W. Acoustic aspects of the aging voice. J Gerontol. 1972;4:119-26.

10. Duchin AW, Mysak ED. Disfluency and rate characteristics of young adult, middle-aged, and older males. J Commun Disord. 1987;20:245-57.

11. Caruso AJ, McClowry MA, Max L. Age-related effects on speech fluency. Semin Speech Lang Hear 1997;18:17180 .

12. Searl JP, Gabel RM, Fulks JS. Speech disfluency in centenarians. J Commun Disord 2002;35:383-92.
13. Yairi E, Clifton NF. Disfluent speech behavior of preschool children, high school seniors, and geriatric persons. J Speech Hear Res. 1972;15:714-9.

14. Manning WH, Monte KL. Fluency breaks in older speakers: implications for a model of stuttering throughout the life cycle. J Fluency Disord. 1981;6:35-48.

15. Preti D. A linguagem dos idosos: um estudo da análise da conversação. São Paulo: Contexto; 1991.

16. Leeper LH, Culatta R. Speech fluency: effect of age, gender and context. Folia Phoniatr Logop. 1995;47:1-14.

17. Riley GD. Stuttering severity instrument for children and adults. Austin: Pro-Ed; 1994.

18. Medical Subject Headings (Index Medicus) [acesso 2007 março 28]. www.nlm.nih.gov/mesh/meshhome.htm.

19. Schneider EL, Guralnik JM. The aging of America: impact on health care costs. J Am Med Assoc 1990;263:2354-5.

20. Ramos LR. Fatores determinantes do envelhecimento saudável em idosos residentes em centro urbano: Projeto Epidoso, São Paulo. Cad Saúde Pública. 2003;19(3):793-8.

21. Ramos LR, Simões E, Albert MS. Dependency on daily living and cognitive impairment strongly predicted mortality among urban aging residents in Brazil: a two-year followup. J Am Geriatr Soc. 2001;49:1168-75.

22. Zackiewicz DV. Avaliação quantitativa e qualitativa das disfluências em indivíduos gagos e fluentes. [Dissertação de Mestrado]. São Paulo; Faculdade de Medicina da Universidade de São Paulo. 1999.

23. Martins VO, Andrade CRF. A ocorrência de pausamento na fala de idosos: estudo da frequência e da duração. Rev Soc Bras Fonoaudiol. 2005; Suplemento Especial, XIII Congresso Brasileiro de Fonoaudiologia.

24. Andrade CRF, Juste F. Aplicação de um teste americano de severidade da gagueira (SSI) em crianças fluentes falantes do Português Brasileiro. Pró-Fono 2001;13(2):177-80. 